\title{
A SOUTHERN HEMISPHERE MILLIMETRE ARRAY
}

\author{
R.S. BOOTH \\ Onsala Space Observatory \\ Chalmers Universirty of Technology \\ S-43992 Onsala,Sweden.
}

\section{INTRODUCTION}

The southern night sky is a sight of overwhelming beauty. It is hard to forget one's first view of the night sky from La Silla with the Galactic centre overhead and the brilliant constellations of Sagittarius and Scorpius with their bright stars and copious amounts of dust. In fact for a millimetre astronomer, it is the dust that is so striking and one feels for the first time that one is seeing tangible visual evidence of the intriguing molecular clouds that dominate our discipline.

Ironically it is the observation of these southern molecular clouds that have shaped much of millimetre radio astronomy, yet we persist in observing them from the northern hemisphere through many air masses of atmospheric attenuation.

Having built the Swedish ESO Submillimtre Telscope, SEST in the south, on La Silla, in Chile, Swedish and other European astronomers would like to follow up this initiative with a southern hemisphere millimetre array, taking advantage of the excellent dry mountain sites. There is much to be done with such an instrument.

\section{WHY A SOUTHERN MILLIMETRE ARRAY?}

All of the currently used millimetre arrays have been built in the northern hemisphere, generally because they have been developed at established radio observatories (Berkeley, Caltech and Nobeyama) or they are within easy access of centres of population (Plateau de Bure). Only the Australia Telescope, which will be useful down to a wavelength of $3 \mathrm{~mm}$ is on a southern site. Yet many of the formative observations of millimetre astronomy are of low (negative) declination sources ( the Galactic centre, Ophiucus, Orion, etc) and their observation by interferometry is hampered by atmospheric phase noise and poor baseline coverage. Clearly we need a purpose built millimetre interferometer on a southern hemisphere site. 
Optical astronomers got their act together many decades ago with observatories in for example South Africa and more than a quarter of a century ago.the South American sites were developed by AURA (Cerro Tololo) and ESO (La Silla). With the large telescopes established on these sites and with the AAT, and of course the radio telescopes of the Australia Telescope National Facility, and now SEST, a catalogue of unique southern objects has been built up.

\section{IMPORTANT SOUTHERN OBJECTS FOR MILLIMETRE INTERFEROMETRY}

Sites of star formation

We know that four out of five of the nearest sites of low mass star formation are in the southern hemisphere. These can be detailed with reference to the compilation of papers by Reipurth (1991):

$\begin{array}{lll}\text { Chamaeleon } & \text { Declination }-77^{\circ} & \text { Distance } 140 \mathrm{pc} \\ \text { Lupus } & \text { Declination }-40^{\circ} & \text { Distance } 150 \mathrm{pc} \\ \text { Corona Australis } & \text { Declination }-37^{\circ} & \text { Distance } 130 \mathrm{pc} \\ \text { Ophiuchus } & \text { Declination }-24^{\circ} & \text { Distance } 160 \mathrm{pc}\end{array}$

and we also find other such regions a little further away the Serpens region at $310 \mathrm{pc}$, Norma, somewhere between 500 and $700 \mathrm{pc}$ and the Gum nebula in the Puppis and Vela region at about $450 \mathrm{pc}$. Even Orion is easily observed from southern sites.

\section{The Galactic Centre}

We have in this meeting had a fascinating insight into the observations of the Galactic centre. However, most millimetre maps are made with highly elliptical beams doing poor justice to the real structure. Seen from Chile, the Galactic centre passes almost overhead, and the interferometer beam would be beautifully circular giving much improved images,

The Magellanic Clouds.

The Magellanic clouds, LMC ans SMC, at distances of 50 and $60 \mathrm{kpc}$ respectively are the nearest galaxies. They are of great interest to millimetre astronomers because they are low metallicity systems undergoing star formation, and e.g. observations of their $\mathrm{CO}$ emission can be used to test the universality of the CO to molecular hydrogen ratio. SEST observations (e.g. Johansson, 1990; Rubio, 1990) have shown that their CO emission is clumpy and often unresolved with the SEST beam. High resolution interferometric observations should reveal a wealth of detail in many clouds. Of special 
interest is the 30 Dor region, a possible region of sequential star formation.(see Booth, 1992)

\section{Other galaxies}

The southern sky contains several grand design spiral galaxies of interest to millimetre astronomers, e.g. M 83 (Wiklind et al, 1990), many interesting interacting systems, e.g. NGC 3256 (Aalto et al, 1991), and the unique nearby, double lobed radio galaxy Centaurus $\mathrm{A}$ which shows $\mathrm{CO}$ in absorption against the nucleus.( Israel et al, 1991; Eckart et al, 1990). High resolution observations of the strong molecular emission in these objects and other bright southern galaxies will add considerably to our knowledge of star formation and star bursts in galaxies.

\section{CONCEPTS FOR A SOUTHERN ARRAY.}

The first Swedish concept for a southern array was presented by the author at a special session on millimetre interferometry following a SEST user's meeting in May, 1991, at ESO headquarters, Garching. This was an array of $10 \times 8 . \mathrm{m}$ antennas to be built near the ESO VLT site on Cerro Paranal, in Northern Chile. The idea, following the success of SEST, was to capitalise on the ESO infrastructure development to keep costs as low as possible. It was estimated that the array would cost about 50 million US dollars.

At the ESO meeting there was considerable enthusiasm for a southern array and a working group was set up, under the chairmanship of the author, to discuss the 'Swedish', and alternative concepts. The membership of that group is as follows:

R.S. Booth (Onsala,Chairman), L. Bååth (Onsala), P. Dewdney (Penticton, Canada) D. Downes (IRAM), M, Grewing (IRAM), S. Guilloteau (IRAM), F. Israel (Leiden), P. Shaver (ESO), T. Wilson (MPI, Bonn). M. Ishiguro (NRO) was unable to attend.

Before the group met, in December, 1991, the discovery of $\mathrm{CO}$ in the high redshift $(z=2.286)$ galaxy, IRAS $10214+4724$, about which we have heard so much in this meeting, was announced by Brown and Vanden Bout (1991). This changed entirely the mode of thinking for many of us. The concept that emerged from the meeting was for a very large millimetre array, capable of early universe studies using red-shifted $\mathrm{CO}, \mathrm{CI}$, etc., and even continuum emission from dust, to be placed on the best possible site.

\section{A LARGE SOUTHERN MILIMETRE ARRAY}

I now present a concept of the large array for the southern hemisphere. This is largely based on suggestions of the working group, and in particular those of D. Downes. 
The scientific potential of the new array should be such that as well as providing a quantum jump in the 'standard' millimetre science, it should be capable of opening up new directions. Thus we see the scientific goals as follows:

-early universe studies on what will be a population of distant galaxies, with relatively weak, red-shifted $\mathrm{CO}$ signals.

-high resolution studies of proto plantary discs and protostars

-high resolution studies of evolved stars

-deep and detailed observations of nearer galaxies and the Magellanic Clouds.

-millimetre continuum observations of quasars and radio galaxies.

In order to achieve many of these goals the interferometer should be capable of achieving a resolution of 0.1 arcsec at $2.6 \mathrm{~mm}$ wavelength. This implies baselines of $10 \mathrm{~km}$ and as a consequence, high sensitivity.

The ideal new instrument would therefore provide a significant ( $x 10)$ improvement over existing, or planned millimetre telescopes The IRAM $30 \mathrm{~m}$ antenna has a collecting area of order $700 \mathrm{~m}^{2}$; the MMA will have $1800 \mathrm{~m}^{2}$. It is suggested that a 'next generation' instrument should have a collecting area of at least $7000 \mathrm{~m}^{2}$.

The goals could be achieved with an array of $40 \times 15$ m telescopes, or $90 \times 10$ m telscopes.

\section{EARLY UNIVERSE STUDIES}

It is interesting to elaborate on this particular new goal for millimetre astronomy. The detection of the red shifted $\mathrm{J}=3-2$ line of $\mathrm{CO}$ in the IRAS galaxy $10214+4724$ (Brown and Vanden Bout, 1991) and the subsequent detections of $\mathrm{CO}(4-3)$ and $\mathrm{CO}(6-5)$, the $\mathrm{CI}$ line and even thermal continuum dust emission, gives millimetre astronomy a whole new dimension. It shows that millimetre astronomy is a powerful tool for studying dust emission and atomic and molecular line emission at epochs close to those of galaxy formation, their first star bursts and production of heavy elements. Of huge significance is that we do not need to go beyond the millimetre band for such work because of the nature of the CO rotational ladder and similar sequences of lines in other molecules. There is always likely to be a line shifted to he millimetre band no matter what the red shift. 
This makes the millimetre band a much more powerful regime for early universe studies than the $21 \mathrm{~cm}$ line of neutral hydrogen. At high $\mathrm{z}$, the $21 \mathrm{~cm}$ line is shifted down to the $200-500 \mathrm{Mhz}$ band with all its drawbacks.

-There is too much man made interference and too much radio noise from the Galaxy.

-There are problems with phase noise on the interferometer due to the ionosphere and solar bursts, and

-the available bandwidth is so small that sensitivity will be severely

limited.

In contrast there is an exciting future here for millimetre astronomy.

\section{SOUTHERN SITES}

Finally a word about sites for a large millimetre array. It is by now well known that the Atacama desert on the coastal side of the Andes, in Northern Chile, contains many large, flat tracts of land at altitudes even as high as $5000 \mathrm{~m}$. These sites are extremely dry, with values of precipitable water in the 1 to $1.5 \mathrm{~mm}$ range during large fractions of time. Several apparently excellent accessible sites have been identified recently by Japanese and Swedish exploration teams, and 22 potential locations were found by Raffin and Kusunoki (1992) in a search for submillimetre interferometer sites.

Extensive water vapour measurements have been made only on Cerro Paranal (altitude $2650 \mathrm{~m}$ ), the site chosen by ESO for the VLT project. These show that for at least $30 \%$ of the time between April and November, the atmospheric precipitable water vapour content is below $1.5 \mathrm{~mm}$; it is below $3 \mathrm{~mm}$ for $60 \%$ of the same period. (see for example the reports by Sarazin, 1990 and Martin, 1991). Paranal is only $15 \mathrm{~km}$ from the ocean; the higher inland sites probably have a lower integrated atmospheric water vapour content.

All of these factors suggest that a site in Northern Chile would be an ideal location for the large millimetre array.

\section{CONCLUSION}

It has been argued that millimetre astronomers should consider building a large millimetre array as a 'next generation' instrument in Northern Chile, with its excellent sites and access to the southern sky, The array should represent a major step in millimetre astronomy should have a collecting area in the range 7000 to $10000 \mathrm{~m}^{2}$ with baselines up to $10 \mathrm{~km}$. Such an instrument is inspired by the exciting potential of millimetre astronomy for early universe studies but would revolutionise millimetre astronomy in general. The cost of such a venture is high; we estimate at least $\$ 250 \mathrm{M}$. The only way to achieve such a goal is therefore by international collaboration. I paraphrase Ishigurosan's words - lets get together and do it! 


\section{REFERENCES}

Aalto, S., Black, J.H, Booth, R.S., Johansson, L.E.B. 1991, Astron. Astrophys. 247, 291.

Brown, R.L., Vanden Bout, P. 1991. Astr. J, 102, 1956.

Booth, R.S., 1992. in 'New Aspects of Magellanic Cloud Research', eds G. Klare and J. Lequeux, Springer-Verlag, Berlin, in press.

Eckart, A., Cameron, M., Genzel, R., Jackson, J.M., Rothermel, H., Stutzgi, J., Rydbeck, G., Wiklind, T. 1990, Astrophys.J., 365, 522.

Israel, F.P., vav Dishoek, E.F., Baas, F, de Graauw, T., Phillips, T.G. 1991. Astron. Astrophys., 245, L 13.

Johansson, L.E.B. 1991. in 'Dynamics of Galaxies and their Molecular Cloud Distributions', eds F. Combes and F. Casoli, Kluwer, Dordrecht

Martin, R.N. 1990. SMT Technical Memo. UA-90-2 (U. Arizona, Tucson)

Raffin, P., Kusunoki, A. 1992, SAO Submillimetre Array Tech. Memo, No 59.

Reipurth, B. 1991 ESO Scientific Rep. No. 11.

Rubio, M. 1991, in 'Dynamics of Galaxies and their Molecular Cloud Distributions', eds. F. Combes and F. Casoli, Kluwer, Dordrecht.

Sarazin, M. 1990. ESO VLT Site selection Working Group, Final Rep. No. 62.

Wiklind, T., Rydbeck, G, Hjalmarson, A, Bergman, P. 1990, Astron. Astrophys, 232, L11. 